\section{Reduced Background Expression and Improved Plasmid Stability with pET Vectors in BL21 (DE3)}

BioTechniques 29:1234-1238(December 2000)

\begin{abstract}
The T7 polymerase-based pET System ${ }^{\mathrm{T}}$ is one of the most powerful and widely used prokaryotic expression systems available today. Expression of even slightly toxic gene products in BL21 (DE3), however, has been problematic due to basal expression, which leads to decreased plasmid stability and variable yields following large-scale growth and induction. Use of host strains such as BL21 (DE3) pLysS provides high stringency and consistent expression but typically at the cost of reduced protein levels upon induction. The experiments presented here suggest that catabolite repression can effectively reduce basal expression of the T7 polymerase gene in BL21 (DE3), yielding tight regulation and consistency comparable to that of BL21 (DE3) pLysS. By switching to a poor carbon source for the final growth cycles, the higher expression levels typical of BL21 (DE3) can readily be obtained upon induction.
\end{abstract}

\section{INTRODUCTION}

Since the introduction of T7 polymerase-based expression (i.e., the pET System $^{\mathrm{TM}}$; Novagen, Madison, WI, USA), thousands of homologous and heterologous proteins have been successfully expressed to very high levels in E. coli strain, BL21 (DE3) (9). Aside from the unavoidable limitations of prokaryotic expression, the single greatest problem associated with this system has been the difficulty of expressing toxic or even slightly toxic gene products, due to background expression (8). Background expression that potentially impacts on organismal fitness encourages the selection of bacterial cells that no longer produce high levels of the target protein [i.e., by mutations affecting either the amount or activity of the target protein (10) or potentially the $\mathrm{T} 7$ polymerase itself]. This decrease in plasmid/clone stability ultimately results in highly variable expression when cultures are expanded for large-scale production (8). Co-expression of the T7 lysozyme gene [BL21 (DE3) pLysS] was shown to markedly reduce background expression by binding to, and inhibiting, the low levels of T7 polymerase present before induction (8). Unfortunately, T7 lysozyme was also found to reduce expression following induction, resulting in markedly lower yields in some cases of the target protein (8).

In both BL21 (DE3) and BL21 (DE3) pLysS, T7 polymerase is produced from a chromosomal copy of the T7 polymerase gene (as part of the lambda DE3 lysogen) under the control of the lacUV5 promoter (9). lacUV5 (L8) is a variant of the lac promoter bearing a point mutation in the -10 region $(\mathrm{G} \rightarrow \mathrm{A}$ at -9$)$, which reduces its de- pendence on cyclic adenosine monophosphate/catabolite gene activator protein (cAMP/CAP) complex to initiate transcription (5). This allows induction of these strains even in the presence of a good carbon source (such as glucose-often used for ${ }^{13} \mathrm{C}$ labeling), which reduces intracellular cAMP levels (i.e., catabolite repression). Grossman et al. (4), however, demonstrated that elevation of cAMP levels, by addition of exogenous cAMP or acetate, could lead to increased basal expression of lac promoters and consequently to instability of $\mathrm{pET}$ vectors. In the studies below, the effect of glucose on the basal expression level of the lacUV5 promoter is investigated using T7 polymerasebased expression of a modified green fluorescent protein (GFP).

\section{MATERIALS AND METHODS}

Plasmid pET28b and competent

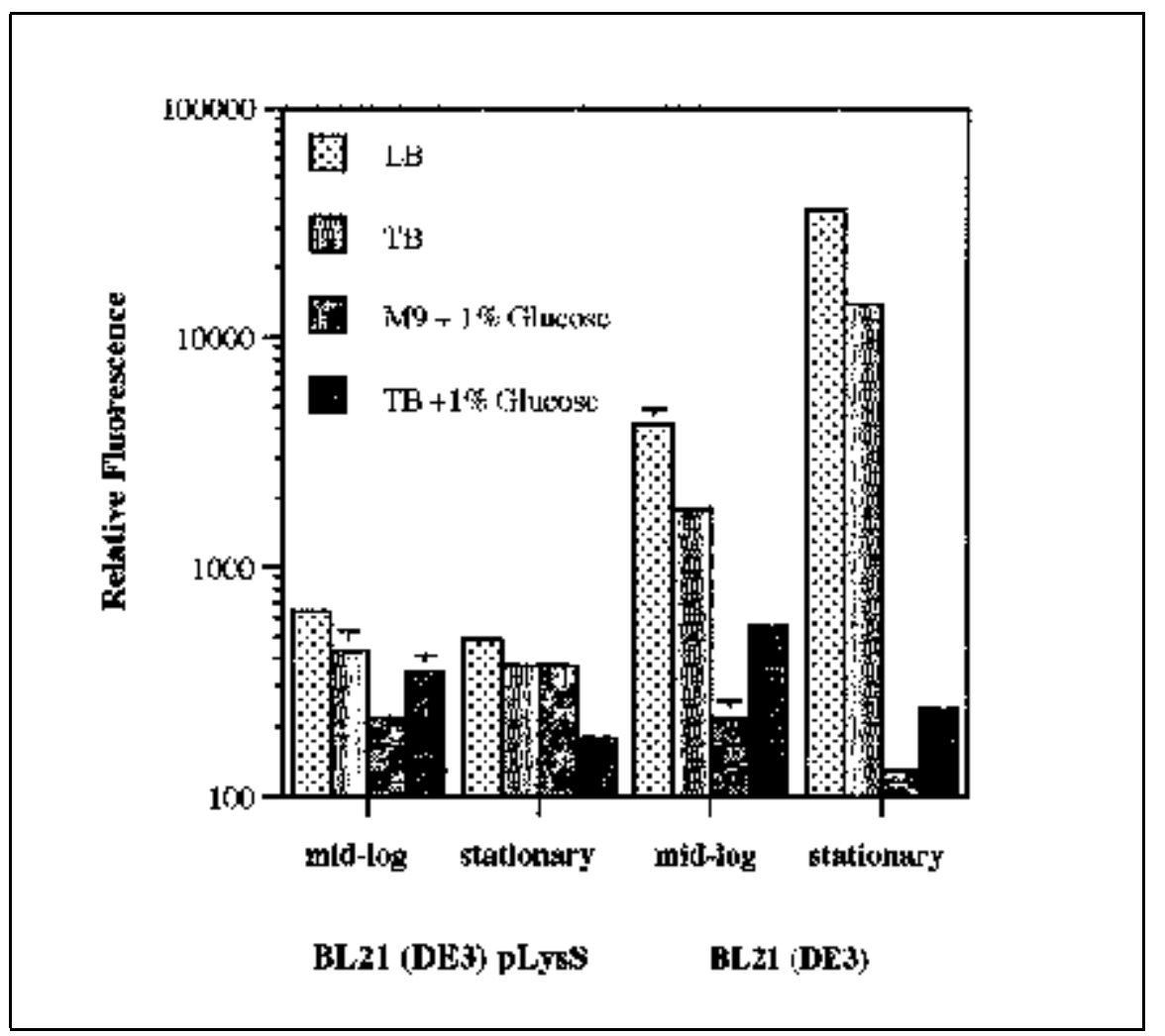

Figure 1. Background expression of GFPuv in BL21 (DE3) pLysS and BL21 (DE3) at 37 ${ }^{\circ} \mathrm{C}$. GFPuv was cloned behind the T7 promoter of pET 28b as described in Materials and Methods. Triplicate cultures were grown, and samples were retrieved during exponential growth (mid-log, 0.5-1.0 $\mathrm{A}_{600}$ ) and shortly after reaching stationary phase. One $A_{600}$ of each culture was harvested by centrifugation. The cleared lysates were evaluated in parallel using a 96-well fluorescence plate reader (see Materials and Methods). The error bar depicts the standard deviation of the triplicate cultures. 
Table 1. Effect of Glucose on Plasmid Stability in BL21 (DE3)

\begin{tabular}{|lccc|}
\hline & $\begin{array}{c}\text { TB } \\
(\mathbf{c f u} / \mathrm{mL})\end{array}$ & $\begin{array}{c}\text { TB with 1\% Glucose } \\
(\mathbf{c f u} / \mathbf{m L})\end{array}$ & $\begin{array}{c}\text { BL21 (DE3) pLysS } \\
(\mathbf{c f u} / \mathrm{mL})\end{array}$ \\
\hline Without Kanamycin & $(3 \pm 1) \times 10^{8}$ & $(3 \pm 1) \times 10^{8}$ & $(6 \pm 1) \times 10^{8}$ \\
With Kanamycin & $(4 \pm 1) \times 10^{5}$ & $(2 \pm 1) \times 10^{8}$ & $(7 \pm 2) \times 10^{8}$ \\
Standard deviation of triplicate plates. & & \\
\hline
\end{tabular}

BL21 (DE3) and BL21 (DE3) pLysS were obtained from Novagen, and plasmid pGFPuv was obtained from Clontech Laboratories (Palo Alto, CA, USA). PCR primers (5'-GATATAGAATTCGCTGGTGCCGCGCGGCAGCCTGC AGATGAGTAAAGGAGAAGAAC-3' and 5'-GATATAAAGCTTTTATTTGTAGAGCTCATCCATG-3') were synthesized and polyacrylamide gel electrophoresis (PAGE)-purified by Genosys (Woodlands, TX, USA). Pfu DNA polymerase was purchased from Stratagene (La Jolla, CA, USA); restriction endonucleases and T4 DNA ligase were purchased from New England Biolabs (Beverly, MA, USA). DH5 $\alpha^{\mathrm{TM}}$ competent cells and complex media compo- nents were obtained from Life Technologies (Rockville, MD, USA).

\section{Plasmid Construction}

The reporter plasmid, pET-GFPuv, was obtained by subcloning a PCR fragment bearing the GFPuv gene from pGFPuv $(1,6)$ into the EcoRI/HindIII sites of pET28b to yield the in-frame fusion with the (His) 6 -thrombin cleavage site leader sequence. DH5 $\alpha$ transformants were obtained following the manufacturer's protocol. The DNA sequence of the construct was confirmed by dideoxysequencing (Bioserve Biotechnologies, Laurel, MD, USA). New ly transformed BL21 (DE3) and BL21

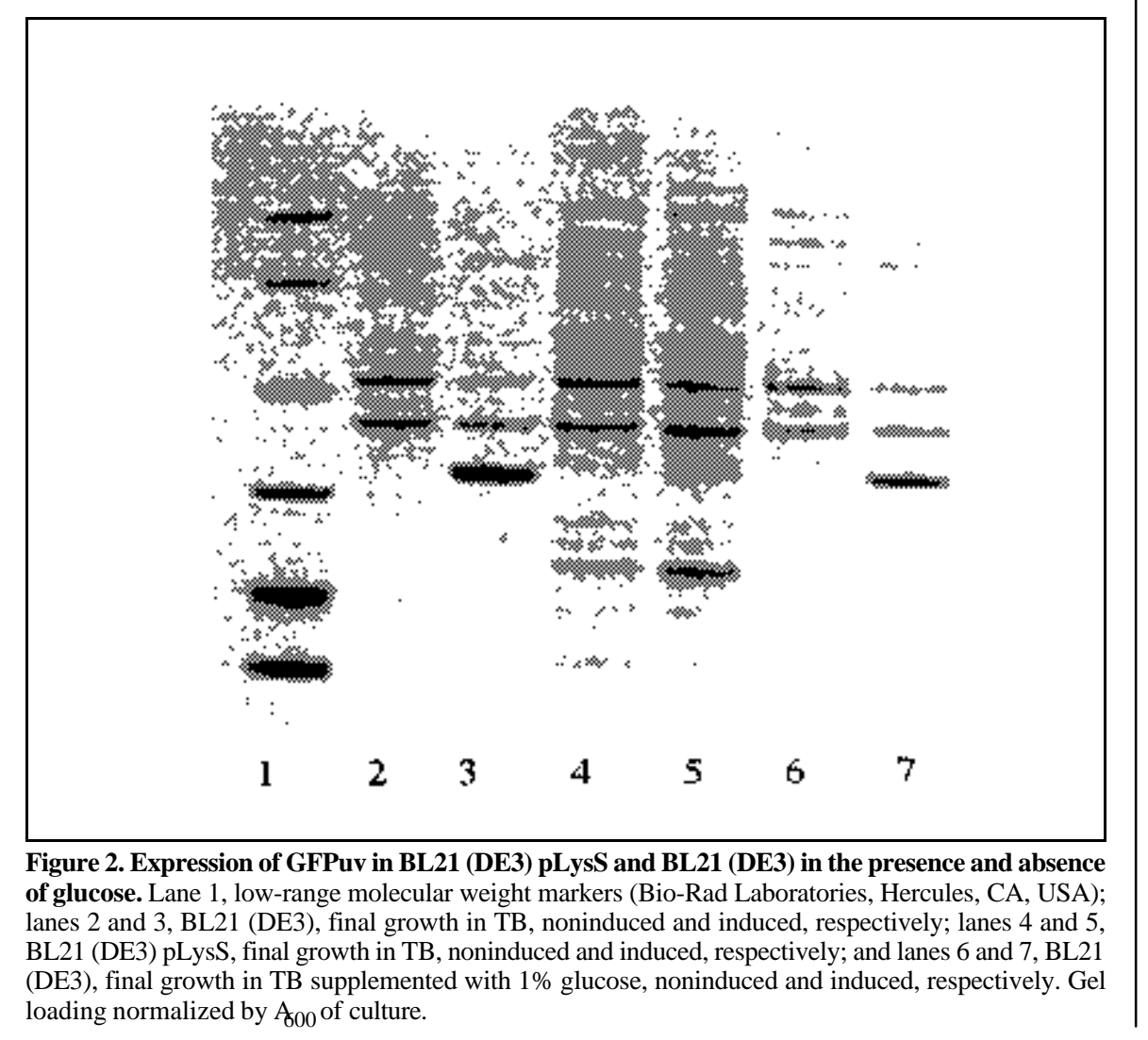




\section{Short Technical Reports}

(DE3) pLysS were used for all studies, and distinct single colonies with normal morphology were chosen at random for inoculation.

\section{Growth and Analysis}

LB seed cultures grown to mid-log ( $\mathrm{A}_{600}$ at approximately 0.7 ) were used to inoculate triplicate cultures for analysis (inoculum 1\% v/v). M9 minimal medium and Terrific Broth (TB) (7), when indicated, were supplemented with $1 \%$ glucose. All media contained $25 \mu \mathrm{g} / \mathrm{mL}$ kanamycin, and 34 $\mu \mathrm{g} / \mathrm{mL}$ chloroamphenicol were added to media for BL21 (DE3) pLysS. Cultures were grown at both $37^{\circ} \mathrm{C}$ and $16^{\circ} \mathrm{C}$ with vigorous shaking and sam pled during exponential growth $\left(\mathrm{A}_{600}\right.$ $0.5-1.0)$ and stationary phase. Control cultures were induced at $A_{600}=0.7$ by the addition of isopropyl- $\beta$-D-thiogalactoside (IPTG) (1 mM final concentration) for $4 \mathrm{~h}$. GFP levels (based on fluorescence and SDS-PAGE analy- sis) were greater than $3 \log$ sigher than basal levels (data not shown).

Following measurement of culture turbidity at $600 \mathrm{nM}$, samples representing 1 A $_{600}$ were harvested by centrifugation, and the cell pellet was resuspended in $0.3 \mathrm{~mL}$ bacterial protein extraction reagent (BPER) lysis solution (a proprietary nonionic detergent solution; Pierce Chemical, Rockford, IL, USA) and incubated at room temperature for $30 \mathrm{~min}$ to ensure complete lysis. The lysates were cleared by centrifugation at $14000 \times \mathrm{g}$ for $30 \mathrm{~min}$ in a microcentrifuge to remove cell debris, and $200 \mu \mathrm{L}$ were transferred to a 96well plate. An MRX fluorescence plate reader (Dynex Technologies, Chantilly, VA, USA) was then used to estimate GFPuv present $\left(\lambda_{\mathrm{ex}}=380 / 20 \mathrm{nM}, \lambda_{\mathrm{em}}=\right.$ $508 / 20 \mathrm{nM}$ ). Background fluorescence from $\mathrm{pET}$-28b transformed BL21 (DE3) and BL21 (DE3) pLysS cultures ( $1 \mathrm{~A}_{600}$ grown in the corresponding medium and processed in an identical fashion) was subtracted from corresponding experimental readings.

Plasmid stability in BL21 (DE3) was checked by serially passaging ( $1 \%$ $\mathrm{v} / \mathrm{v}$ inocula) seed cultures $\left(37^{\circ} \mathrm{C}\right.$, overnight) grown in TB with and without supplemental glucose (1\%) to simulate expansion of seed stocks for large-scale fermentations. After three transfers, in the absence of kanamycin [note: for BL21 (DE3) pLysS, TB was supplemented with chloroamphenicol only], cultures were plated on LB plates with and without kanamycin to estimate the fraction of bacteria that had retained the expression plasmid.

To confirm that good protein expression levels could still be obtained from BL 21 (DE3) cultures propagated/expanded in TB supplemented with $1 \%$ glucose, an analogous experiment to the one described above for evaluating plasmid stability was conducted, except the media were supplemented with kanamycin. A newly transformed BL 21 (DE3) colony was grown and serially passaged in $\mathrm{TB}$ with $1 \%$ glucose for three days and then used to inoculate $(1 \% \mathrm{v} / \mathrm{v})$ supplemented or nonsupplemented TB. A BL 21 (DE3) pLysS transformant was grown in TB supplemented only with chloroamphenicol (and kanamycin). Both cultures were induced at $A_{600}=$ 0.7 by the addition of IPTG ( $1 \mathrm{mM}$ final concentration) for $4 \mathrm{~h}$. Expression was evaluated by SDS-PAGE.

\section{RESULTS AND DISCUSSION}

The background expression level of GFPuv from the T7 promoter in both BL21 (DE3) and BL21 (DE3) pLysS with various media is shown in Figure $1\left(37^{\circ} \mathrm{C}\right)$. Background expression in BL21 (DE3) pLysS was consistently low, irrespective of the medium, during both exponential growth and stationary phase (Figure 1). Background levels of GFPuv in BL21 (DE3), however, were 4- to 8-fold higher than BL21 (DE3) pLys during exponential growth and 40 - to 80 -fold higher at stationary phase in nonsupplemented LB and TB. In contrast, basal levels of GFPuv in BL21 (DE3), when grown in M9 supplemented with $1 \%$ glucose, were equal to those of BL21 (DE3) pLysS, in both exponential and stationary phase. Upon supplementing $\mathrm{TB}$ with $1 \%$ glucose, background leakage also appeared to be suppressed to BL21 (DE3) pLysS levels at early and late time points. A virtually identical result was obtained when cultures were grown at $16^{\circ} \mathrm{C}$ (data not shown).

The reduction of background expression in BL21 (DE3) in the presence of glucose can be easily understood as the effect of catabolite repression on the lacUV5 promoter, which drives the $\mathrm{T} 7$ polymerase gene in the DE3 lysogen (4). Prior studies of lacUV5 suggest that its affinity for the cAMP/CAP activator complex has not been totally abolished but merely reduced by 5 - to 10 -fold (3). Formation within the cell of cAMP/CAP activator complexes, due to the absence of a good carbon source, should therefore activate, to some extent (despite its reduced affinity and the binding of the lacI gene product to the lac operator), the lacUV5 promoter and increase production of T7 polymerase (2,3). These additional $\mathrm{T} 7$ polymerase molecules, once formed, would repeatedly initiate transcription from the episomal T7 promoters, thus multiplying the effect and generating the observed GFPuv. In stationary phase cultures, where only the poorest carbon sources remain, activation/leakage of the lacUV5 promoter would be the most pronounced. 
The impact of promoter leakage on clone/plasmid stability, when expressing even slightly toxic proteins, has been well established (8). In Table 1, it can be seen that repeated growth of BL21 (DE3) in nonsupplemented TB clearly leads to plasmid loss compared with growth in the presence of $1 \%$ glucose or in the presence of the pLysS plasmid. It may be inferred that even in the presence of antibiotics, promoter leakage from growth on nonsupplemented media would lead to increased selection pressure, which would lead to ever more variable results when cultures were finally induced (10). Heretofore, the most common remedy has been to tighten regulation through the constitutive expression of the T7 lysozyme gene [i.e., BL21 (DE3) pLysS] (8). Unfortunately, these same lysozyme molecules can cause spontaneous bacterial lysis during growth and continue to inhibit the polymerase following induction, resulting in reduced expression levels (8).

Unlike the constitutive expression of T7 lysozyme, however, catabolite repression can be easily eliminated by simply conducting the final growth, before induction, in a poor carbon source medium such as LB, TB or M9 with glycerol. In Figure 2, GFPuv expression is compared in BL21 (DE3) with and without glucose in the final growth medium. A seed culture propagated in TB supplemented with $1 \%$ glucose was used to inoculate both cultures (1\% $\mathrm{v} / \mathrm{v})$. For comparison, expression in BL21 (DE3) pLysS is shown. Clearly, expression is better using BL21 (DE3), and significant improvement is seen simply by removing the glucose from the final growth/induction medium. By transforming, plating and subculturing in the presence of a good carbon source and then expressing in a nonsupplemented medium, BL21 (DE3) can provide the tight regulation and clone stability associated with BL21 (DE3) pLysS without the potential for autolysis and reduced expression (8) associated with this strain.

\section{REFERENCES}

1.Crameri, A., E.A. Whitehorn, E. Tate and W.P. Stemmer. 1996. Improved green fluorescent protein by molecular evolution using
DNA shuffling. Nat. Biotechnol. 14:315-319.

2.Fried, M.G. and D.M. Crothers. 1983. CAP and RNA polymerase interactions with the lac promoter: binding stoichiometry and long range effects. Nucleic Acids Res. 11:141-158.

3.Fried, M.G. and D.M. Crothers. 1984. Equilibrium studies of the cyclic AMP receptor protein-DNA interaction. J. Mol. Biol. 172:241-262.

4.Grossman, T.H., E.S. Kawasaki, S.R. Punreddy and M.S. Osburne. 1998. Spontaneous cAMP-dependent derepression of gene expression in stationary phase plays a role in recombinant expression instability. Gene 209:95-103.

5.Hirschel, B.J., V. Shen and D. Schlessinger. 1980. Lactose operon transcription from wildtype and L8-UV5 lac promoters in Escherichia coli treated with chloramphenicol. J. Bacteriol. 143:1534-1537.

6.Lissemore, J.L., J.T. Jankowski, C.B. Thomas, D.P. Mascotti and P.L. deHaseth. 2000. Green fluorescent protein as a quantitative reporter of relative promoter activity in $E$. coli. BioTechniques 28:82-89.

7.Sambrook, J., E.F. Fritsch and T. Maniatis. 1989. Molecular Cloning: A Laboratory Manual, 2nd ed., Vol. 3. CSH Laboratory Press, Cold Spring Harbor, NY.

8.Studier, F.W. 1991. Use of bacteriophage T7 lysozyme to improve an inducible T7 expression system. J. Mol. Biol. 219:37-44.

9.Studier, F.W. and B.A. Moffatt. 1986. Use of bacteriophage T7 RNA polymerase to direct selective high-level expression of cloned genes. J. Mol. Biol. 189:113-130.

10.Weisemann, J.M. and G.M. Weinstock. 1985. Direct selection of mutations reducing transcription or translation of the recA gene of Escherichia coli with a recA-lacZ protein fusion. J. Bacteriol. 163:748-755.

Address correspondence to Dr. B.A. Malcolm, Antiviral Therapy, ScheringPlough Research Institute, 2015 Galloping Hills Road, Kenilworth, NJ 07033, USA. email:bruce.malcolm@spcorp.com

Received 17 March 2000; accepted 16 August 2000.

\section{Shi-hao Pan and \\ Bruce A. Malcolm \\ Schering-Plough \\ Research Institute \\ Kenilworth, NJ, USA}

